\title{
Effective Thermal Simulation of Power Electronics in Hybrid and Electric Vehicles
}

\author{
Wenjun Liu, Hauck Torsten and Josef Drobnik \\ ${ }^{l}$ Freescale Semiconductor, 2100 E.Elliot Rd, Tempe,AZ 85284,B35741@freescale.com
}

\begin{abstract}
Thermal management is critical in development of hybrid and electric vehicles since they contain multiple high-density power modules that require compact integration and effective cooling. During operation the power modules generate large amounts of heat leading to significant temperature increases and thermal gradients inside dies and across the packages. Electro-thermal simulation is needed to choose the best operating regime to meet the thermal and electrical requirements. However, this is usually computationally expensive. One effective method is to develop a compact but accurate thermal model to capture the thermal physics that can be used in the system-level electro-thermal model. In this paper an effective Model Order Reduction (MOR) is developed that drastically reduces the number of Degree of Freedoms (DOFs) of the original large-dimension ODE system. Finite Element Analysis (FEA)/ Computational Fluid Dynamics (CFD) simulation is first conducted to find the optimal pin-fin to rear channel ratio and the optimal pin fin shape that gives lowest peak temperature and pressure drop. The convective heat transfer coefficients exacted from FEA/CFD is input to the MOR model. The MOR model is then applied to a converter assembly, and results show that it can reduce the computation time from 2 hours to 1 second with reasonable error compared to the FEA/CFD predictions. The MOR thermal model can be further coupled with electrical circuit models to form a system model to predict the temperature profile and the power modules' electrical transient performance.
\end{abstract}

\section{Introduction}

The power devices Hybrid and electric vehicles (HEV/EV) have gained renewed interest recent years as they provide various environmental, economic and national interest benefits over the conventional internal combustion engine-based vehicles [1]. Among these factors, the lower cost per mileage is an important driver for EVs to gain adoption, growth and popularity over Internal Combustion Engine (ICE)-based vehicles. Currently EVs also face certain challenges, mostly battery-related. For EVs to become commercially competitive, battery modules of high capacity, high power density, high energy conversion efficiency, reliable packaging technology, and effective cooling technique are highly desired [2].

Figure 1 shows the configuration of a typical electric vehicle. Compared to ICE-based counterparts EVs have a much simpler architecture, in which the E-drive (inverter and 
electric motor) and battery packs replace the IC engine and gas tank. The E-drive is independent of the primary source of the electricity, and is the heart of EV/HEV. Among all components, the inverter is a critical module and is always needed between the battery and electric motor(s) in an EV.

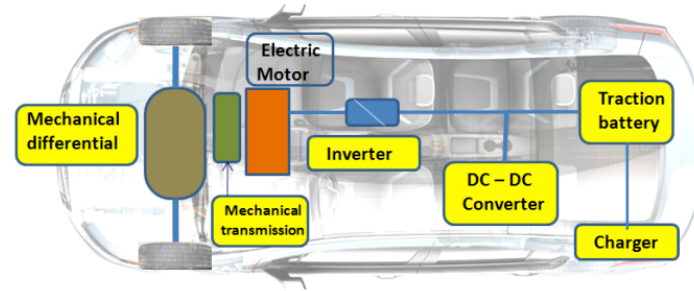

Figure 1: Typical configuration of an electric vehicle

As shown in Figure 1 EVs contains various highpower electronics, e.g., inverter, converter, battery pack and motor, which need effective thermal management. During operation EV power electronics generate large amounts of heat $(\sim 100$ s watts per die), which leads to large heat flux at the die- and package- levels. Therefore effective thermal management must be considered to achieve desired performance and reliability of the power electronics. In power electronics a small percentage of power loss can result in huge heat loss. For example, a $50 \mathrm{~kW}$ converter with $95 \%$ efficiency will generate 2.5 $\mathrm{kW}$ heat. In EV's two major electric energy losses exist: (a) switch loss of IGBTs or MOSFETs, which can be minimized by better switching performance; and (b) conduction loss, which can be minimized by reducing device forward drop. Since the environmental temperature changes from season to season and even from day to night, heat generation in EVs power electronics is dynamic and frequencydependent, as shown in Figure 2.

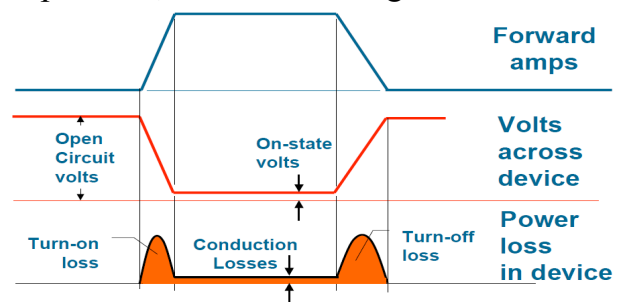

Figure 2: Power loss in EV power electronics [2]

\section{Packaging and cooling}

The power devices The EV's power electronics must offer optimized performance, reliability and cost effectiveness. The current trend of $\mathrm{EV}$ power electronics design is a more integrated and modular design scheme: (a) easy integration, (b) low thermal resistance, (c) low electrical parasitic parameters, and (d) low manufacturing costs. The smaller electrical parasitic parameters not only improve the system conversion efficiency, but also reduce use of the semiconductor dies by reducing both voltage and current rate. A fully integrated system is beneficial in terms of lower cost, simplicity of the cooling system (sharing the existing cold plate), less connectors and wiring and smaller size/weight. It also helps by reducing the delay and power consumption of interconnects.

Figure 3 shows an integrated inverter assembly with three sections inside a metal casting [3]. The control section integrates a micro controller with transceivers. The driver board holds the IGBT or MOSFET drivers as well as motor position and temperature sensors. The driver section drives the IGBTs or MOSFETs, isolates the electric drive and control board, and also performs signal acquisitions, diagnosis and safety functions. The power section contains the IGBTs or MOSFETs power modules and recirculation diodes mounted on a cold plate or heat spreader. It also has sensing of phase voltage/current and temperature measurement capability.

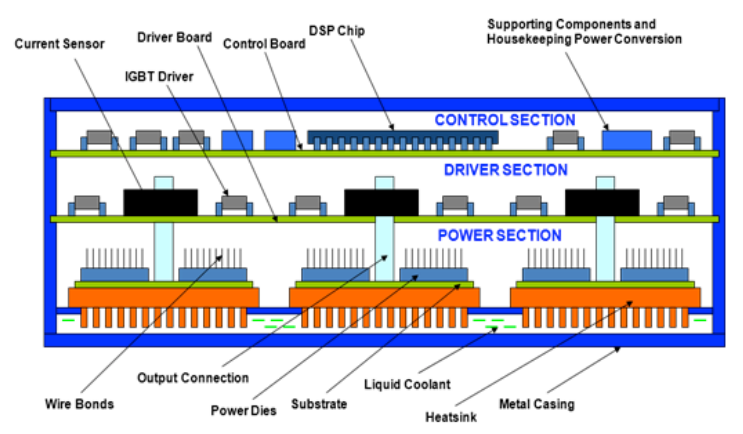

Figure 3: A 3-section inverter assembly inside an enclosure: control section, driver section, and power section.

Various concepts have been pursued for EV power electronics cooling. Early cooling technologies were primarily focused on active/passive air cooling and heat sinks. Liquid cooling has emerged as a promising solution and is actively explored for cooling high-power electronics components, e.g., insulated gate bipolar transistor (IGBT) in inverter/converters. Since the heat transfer coefficient of liquid cooling is about two orders of magnitude higher than that of air cooling, the inverter typically needs liquid cooling given the large power loss $(>1.3 \mathrm{~kW})$. The DC/DC 
converter and charger may be cooled using forced convective liquid or air. For ultra-high power density, two-phase cooling has benefits in cooling rate, size, weight, and power consumption.

Implementation of two-phase cooling methods include improving liquid convective performance using micro- and mini-channel, and using liquid jets/sprays. However, performance of two-phase cooling is difficult to simulate and estimate, making system integration unpredictable or unreliable.

From the thermal point of view, the power section in an inverter assembly (Figure 3), which contains the major heat source, sits directly on the cold plate that has embedded liquid cooling channels. Heat generated in the driver and control sections is mostly dissipated through the highly thermal conductive output connection poles to the cold plate. The module enclosure serves as heat spreader with extended surface. The liquid cooling module (cold plate) in the power section is essentially a liquid-cooled compact heat exchanger, i.e., cold coolant enters the inlet connector, absorbs heat, and leaves the outlet connector taking heat away. The cold plate design in Figure 3 is important in thermal management of EV power devices and needs to be optimized.

\section{FEA/CFD Simulation}

The power devices are main heat generation components in EVs. These power devices dissipate up to $2 \mathrm{~kW}$. As the losses in the inductor/transformer are low, forced air cooling with a simple fan attached to the inductor and transformer is usually adequate. In contrast, most power systems (power devices) in EVs are liquid cooled using a cold plate (e.g., pin fin heat exchanger in Figure 3). Since the cold plate typically takes nearly half of the volume and weight of power converters, its design needed to be optimized for smaller weight and size.

In this section, the liquid cooling of the power modules using a cold plate is modelled by a commercial software package ANSYS CFX [4]. The simulation is a conjugated heat transfer simulation, in which the fluid flow is modelled by Computational Fluid Dynamics (CFD) and the heat transfer by Finite Element Analysis (FEA). To get accurate simulation results, the simulation requires very fine mesh near the structure/fluid interface and across the flow channels, which leads to large computation resources, e.g., long CPU times and large memory usage.
In cold plate design the following design parameters are usually considered: cost, cooling performance, water flow rate, pressure drop, and mechanical strength. Coolant flow rate and pressure drop affects the size of the pumping and plumbing system, and ultimately the cost and physical volume of the coolant distribution system. Therefore in the cold plate design it is highly desired to minimize the coolant flow rates and pressure drop, yet ensuring sufficient thermal performance [3]. A Pin-fin heat sink is chosen because the pin-fin structure drastically increases the total surface area thus heat transfer and has relatively low pressure drop. The cold plate with pin-fin structure is usually used for the power electronics with power densities above 200 $\mathrm{W} / \mathrm{cm} 2$. In this section several cold plates of different pin fin geometry and arrangement were simulated to find the design with highest cooling performance and lowest pressure drop.

\subsection{Pin-Fin to Rear Channel Ratio}

FEA/CFD simulation was first conducted to find the optimal pin-fin to rear channel ratio, i.e., ratio of pin fin length to the flow gap size in the flow direction. Simulation results show that the most effective pin-fin to rear channel ratio corresponding to minimal temperature and pressure increase is $\sim 2: 1-2.5: 1$. Therefore the ratio of $\sim 2.3$ is used in the pin fin heat sink geometry in the subsequent simulations. Figure 4 shows the predicted temperature rise and pressure differential for diamond- and elliptical-shaped pin fin heat sinks.

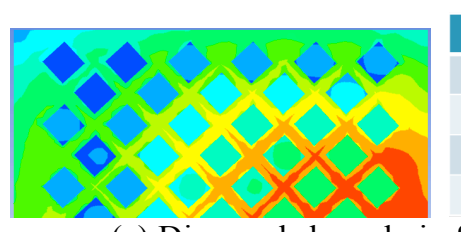

\begin{tabular}{|r|r|r|}
\hline & DT & \multicolumn{1}{l|}{ DP } \\
\hline 2.1 & 38.4 & 196 \\
\hline 2.45 & 33.4 & 192 \\
\hline 2.8 & 36.2 & 185 \\
\hline 3.15 & 37.5 & 172 \\
\hline
\end{tabular}

(a) Diamond shaped pin fin $(l=4 \mathrm{~mm})$

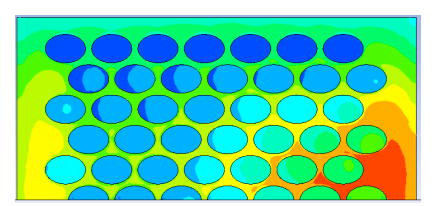

\begin{tabular}{|r|r|r|}
\hline Gap & DT & \multicolumn{1}{|l|}{ DP } \\
\hline 1.75 & 31.4 & 172 \\
\hline 2 & 31.1 & 169 \\
\hline 2.25 & 29.5 & 167.8 \\
\hline 3 & 33.2 & 154 \\
\hline 3.5 & 33.9 & 154 \\
\hline
\end{tabular}

(b) Elliptical shaped pin fin $(a=3, b=1.75 \mathrm{~mm})$

Figure 4: FEA/CFD simulation to find optimal pinfin to rear channel ratio (symmetric geometry).

\subsection{Thermal and Flow Performance}

Next, four cold plates with different pin fin shapes (round, elliptical, diamond, square, rectangular 
cross sections) were simulated to find the shape with optimal cooling performance and lowest pressure drop. In the simulation the wetted perimeters are set same $\sim 16 \mathrm{~mm}$ for all the pin fin shapes. Detailed dimensions are below: round: $\mathrm{r}=2.55 \mathrm{~mm}$; elliptical: $\mathrm{a}=3 \mathrm{~mm}$, $\mathrm{b}=1.75 \mathrm{~mm}$; diamond: $1=4 \mathrm{~mm}$; square: $1=4 \mathrm{~mm}$; rectangular: $a=4 \mathrm{~mm}, b=4 \mathrm{~mm}$. $1 \mathrm{~kW}$ heat flux is applied on top of the heat sink. Coolant temperature at cold plate inlet is set at room temperature, and 1 atmosphere is assumed as outlet pressure. The coolant flow rate is $4 \mathrm{l} / \mathrm{min}$. The simulation results of different pin shapes were compared to get the optimized design that has the maximal cooling performance (lowest peak temperature) and flow performance (minimal coolant pressure drop).

Figure 5a shows the predicted temperature profile of the diamond-shaped pin fin heat sink. The heat sink dimension is $100 \times 8 \times 10 \mathrm{~mm}$. The coolant flow rate is set $41 / \mathrm{min}$. At the $1 \mathrm{~kW}$ heat load, the predicted average temperature increase is about $26.7^{\circ} \mathrm{C}$.

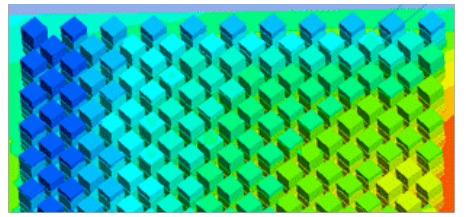

(a) Predicted temperature profile

\begin{tabular}{|c|c|c|}
\hline Gap & $\Delta \mathrm{T}\left({ }^{\circ} \mathrm{C}\right)$ & $\Delta \mathrm{P}(\mathrm{mBar})$ \\
\hline 0.75 & 29.1 & 235.5 \\
\hline 1 & 26.7 & 229.2 \\
\hline 1.25 & 38.5 & 220 \\
\hline
\end{tabular}

(b) Predicted $\Delta T$ and $\Delta p$ at different gap

Figure 5: FEA/CFD prediction of the diamondshaped pin fin heat sink (Dimension: $l=2 \mathrm{~mm}$ )

Figure 6 shows the predicted temperature profile of the elliptical-shaped pin fin heat sink. It has the same inlet flow rate $(4 \mathrm{l} / \mathrm{min})$ and the heat sink dimension is $100 \times 30 \times 7 \mathrm{~mm}$. The predicted average temperature increase at the outlet is about $22.4 \mathrm{oC}$. This shows that the elliptical pin shape has lower $\Delta \mathrm{T}$ and $\Delta \mathrm{p}$ than the diamondshaped pin fin heat sink.

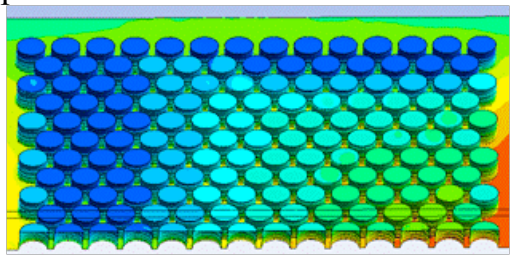

(a) Predicted temperature profile

\begin{tabular}{|c|c|c|}
\hline Gap & DT $\left({ }^{\circ} \mathrm{C}\right)$ & DP (mBar) \\
\hline 0.75 & 29.1 & 206 \\
\hline 1 & 22.4 & 194 \\
\hline 1.25 & 23.2 & 182.2 \\
\hline 1.5 & 23.4 & 173.3 \\
\hline
\end{tabular}

(b) Predicted $\Delta T$ and $\Delta p$ at different gaps

Figure 6: FEA/CFD prediction of the elliptical shaped pin-fin heat sink (Dimension: $a=1.5$, $\mathrm{b}=0.875 \mathrm{~mm}$ )

Figure 7 summarizes the predicted thermal and fluid performance of heat sink of all four pin fin shapes. It shows that the elliptical-shaped fin structure has the lowest peak temperature and the lowest pressure drop, and is therefore the optimal geometry. However, manufacturing such elliptical pin fin is difficult and expensive. In reality, the diamond-shaped pin fin is preferred due to its reasonable cooling performance and low manufacturing cost. Therefore the diamondshaped pin fin heat sinks are used in the $\mathrm{AD} / \mathrm{DC}$ converter assembly simulation in Section 3.3.

\begin{tabular}{|c|c|c|}
\hline Type & $\begin{array}{l}\text { Peak Temp } \\
\left({ }^{\circ} \mathrm{C}\right)\end{array}$ & $\begin{array}{c}\text { Pressure } \\
\text { Drop (mBar) }\end{array}$ \\
\hline Round & 65 & 100 \\
\hline Elliptical & 50 & 41 \\
\hline Diamond & 69 & 61 \\
\hline Rectangular & 66 & 56 \\
\hline
\end{tabular}

Figure 7: Predicted peak surface temperature and pressure drop for various pin fin shape. (Round: $r=2.55$.; elliptical: $a=3, \quad b=1.75$; diamond: $l=4$; square: $l=4$; rectangular: $a=4, b=4$; unit: $\mathrm{mm}$.)

\subsection{Simulation of AC/DC Converter Assembly}

Transient heat transfer of an AC/DC converter assembly with diamond-shaped pin fin heat sinks was also simulated by ANSYS CFX. In the simulation 200 and $250 \mathrm{~W}$ thermal load are applied to heat source (HS) one and two respectively. 20 $\mathrm{W}$ is applied to HS 3 and 4 . A $41 / \mathrm{min}$ water flow rate is applied at the coolant inlet. Coolant temperature at inlet is set at room temperature, and outlet pressure of the cold plate is set to $1 \mathrm{~atm}$. At time zero, the assembly is at room temperature. Figure 8 shows the model geometry and the predicted temperature field at $\mathrm{t}=2000$ second (already reaching steady state). The temperature at four heat source locations, e.g., HS1, HS2, HS3, and HS4, will be compared to MOR results in Figure 11. 


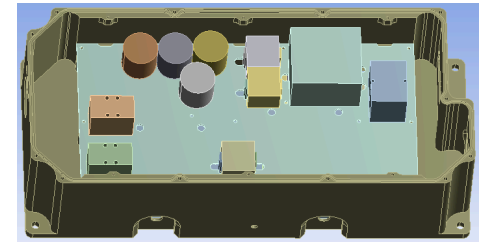

(a) $\mathrm{AC} / \mathrm{DC}$ converter model

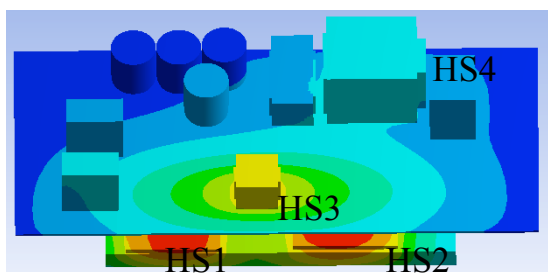

(b) Predicted temperature field at $t=2000 \mathrm{sec}$ Figure 8: Geometry and FEA/CFD predicted thermal profile of an AC/DC converter assembly

The FEA/CFD simulation captured the coupling of heat transfer and fluid flow, and can accurately predict the detailed thermal profile and pressure distribution in the entire simulation domain. However the simulation takes much resources and is computationally expensive. For the mesh used ( 20,000 elements), each simulation takes over 2 hours in a Workstation (16G memory, 2.4G Dual-Core CPU). Therefore one goal of the work is to implement the Model Order Reduction (MOR) to reduce computation time while providing reasonable accuracy.

\section{Model Order Reduction (MOR}

Model Order Reduction is a type of fast physics model that simplifies the solution of large problems using drastically reduced numbers of DOFs. The formal Model Order Reduction theory is based on the approximation of the transfer function of the original dynamic system. It has been proved that in the Krylov subspaces the reduced system matches the moments of the original system for the given expansion point [56]. The dimension (or DOFs) of the reduced model during the model reduction process can be controlled by the approximation error specified by the user, i.e., the tradeoff between error and number of DOFs which can be controlled as needed. Usually larger number of DOFs gives higher accuracy (more moment matching), but requires longer computation time.

\subsection{MOR Method}

The MOR method can be combined with commercial finite element software to (a) speed up a transient or harmonic analysis, (b) generate automatically compact models for system-level simulation, and (c) incorporate finite element packages during the design phase. MOR methods based on the Padé approximation are widely used with finite element software. Although it does not have global error estimates, it can use an error indicator in practice and works well for most finite element models [7].

Model reduction typically starts with discretization of governing partial differential equations into dynamic systems of the first order as Eqn. (1). This can be done in a finite element package such as ANSYS.

$$
\begin{gathered}
C \frac{\partial T}{\partial t}-k \frac{\partial^{2} T}{\partial x^{2}}=Q(x, t) \text { into } \\
E \dot{T}=K T+B u(t)
\end{gathered}
$$

To use model reduction, Eqn. (1) is rewritten as:

$$
\begin{aligned}
& E \dot{T}=K T+B u \\
& y=C T
\end{aligned}
$$

where $\boldsymbol{E}$ and $\boldsymbol{K}$ are the system matrices, $\boldsymbol{B}$ is the input matrix, $\boldsymbol{C}$ is the output matrix.

Eqn. (1) differs from (2) in: (a) splitting of the load vector to a product of a constant input matrix $\boldsymbol{B}$ and a vector of input $\boldsymbol{u}$ functions, and (b) introduction of the output vector $\boldsymbol{y}$ that contains some linear combinations of the state vector that are of interest in system level simulation.

After applying model reduction, Eqn. (2) is converted to a low-dimensional approximation in the form:

$$
\begin{gathered}
V^{T} E V \dot{z}=V^{T} A V z+V^{T} B u(t) \\
E_{r} \dot{z}=A_{r} z+B_{r} u(t)
\end{gathered}
$$

Eqn. (3) describes the dependence of the output vector $\boldsymbol{y}$ on the input vector $\boldsymbol{u}$. The dimension of the reduced state vector $\boldsymbol{z}$ is much less than the dimension of the original state vector $\boldsymbol{T}$.

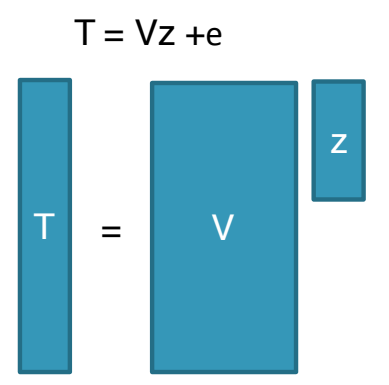

Figure 9: MOR as a projection of the original system onto the low-dimension subspace [8]. 
Model reduction is based on the assumption that the movement of a high-dimension state vector can be well approximated by a lower-dimension subspace. Provided this subspace is known, the original system can be projected on it as shown in Figure 9.

\subsection{MOR Applied to AC/DC Converter Assembly}

The MOR process used in this work is described in Figure 10. First the finite element software (ANSYS) is used to produces a system of ordinary differential equations after the discretization in space the partial differential equation describing the model (consisting of only solid, no liquid). Since the system matrices are high dimensional and sparse, implementation of a model reduction algorithm uses a sparse solver and a storage scheme for sparse matrices to achieve high computation efficiency.

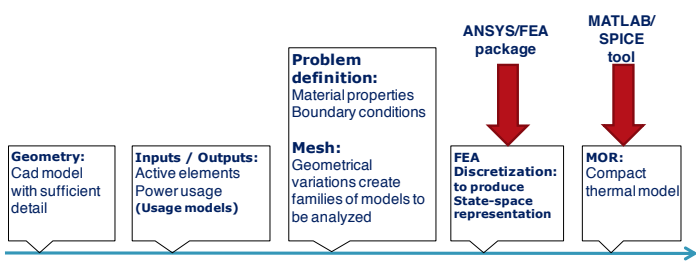

Figure 10: Principle and process of MOR method

To implement model order reduction, special MOR software, MOR for ANSYS [9], was used in this work which reads system matrices from ANSYS FULL files as input, runs a model reduction algorithm, and then writes reduced matrices as output. The convective heat transfer coefficients calculated from FEA/CFD are used as the thermal boundary in MOR model (solid domain only). Note the thermal boundary condition transfer from ANSYS to MOR introduces certain relatively small errors.

The process of generating FULL files in ANSYS CFX is automated through scripting. After the model reduction process, the reduced matrices (output of MOR) can be subsequently processed in MATLAB/Simulink, Spice, Simplorer, or other simulation tools. MATLAB/Simulink was used in this work for the final processing.

For the ease of comparison the MOR process was applied to the converter assembly with diamond shaped pin-fin heat sink that was simulated by FEA/CFD in section 3. The geometry, heat generation loads, and thermal boundary conditions are the same. The convective heat transfer coefficients calculated from FEA/CFD were used as the thermal boundary in the MOR model. The reduced model still has all the heat sources and considers thermal cross talk between individual components. Temperatures were calculated at four heat source locations, e.g., (a) HS1, (b) HS2, (c) HS3, and (d) HS4, of the AC/DC converter with diamond-shaped pin fin heat sinks in Figure 8. The MOR results were then compared to FEA/CFD prediction in Figure 11. Comparison of the results shows that the error between MOR and FEA/CFD is very small for such a large model reduction. For locations HS1 and HS2 the error is $<1 \%$ after reaching steady state. For locations HS3 and HS4, the error is $\sim 3 \%$ after reaching steady state. Before reaching steady state, the error is $<2 \%-6 \%$ for all four locations.

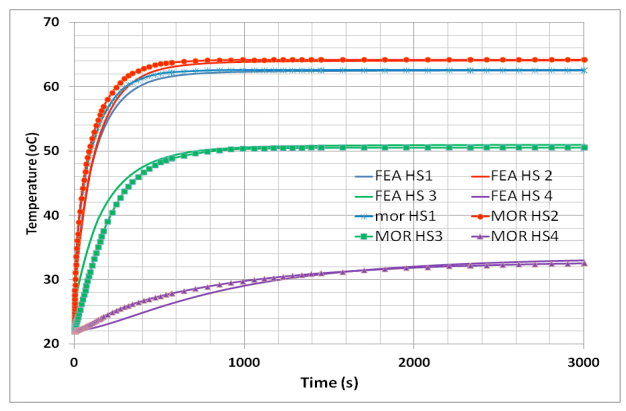

Figure 11: Comparison of FEA/CFD vs. MOR results at four locations of $\mathrm{AC} / \mathrm{DC}$ converter.

The transient time range, number of DOFs, and solution time for the MOR and ANSYS FEA/CFD methods were compared in Table 1 . In the MOR the dimension is reduced to only 180 DOFs, compared to $900 \mathrm{k}$ in ANSYS FEA/CFD. The CPU time for MOR is $\sim 1$ second, while solution time in ANSY is $>2$ hours.

Table 1: Comparison of number of DOFs and CPU time for MOR and FEA/CFD simulation.

\begin{tabular}{l|l|l|} 
& MOR & Ansys (FEM) \\
\hline Transient Time Range [s] & 4000 & 4000 \\
\hline Dimension & 180 (15 X input) & $900 \mathrm{~K}$ DOF \\
\hline CPU time & $1 \mathrm{sec}$ & $>2 \mathrm{hr}$
\end{tabular}

The comparison in Table 1 shows that the MOR method is a good representation of the original large-dimension ODE system (e.g., relatively small error) and is very effective in simplifying the original ODE systems (e.g., drastically reduced solution time). The simplified thermal model by the MOR method can be subsequently coupled to electrical circuit models to predict the power module's thermal profile and electrical performance. 


\section{Conclusion}

Hybrid and electrical vehicles contains highpower density electronics which requires effective thermal management and special packaging design to achieve high reliability and performance. Large-dimension coupled electrothermal simulations are often required for thermal and packaging design of EV power electronics which is usually computationally expensive. This work presented a fast and accurate MOR modelling method to simulate the thermal performance of EV power electronics. FEA/CFD using ANSYS CFX was first conducted to simulate the coupled heat transfer and fluid flow in various pin fin heat sinks. Simulation results show that the most effective pin-fin to rear channel ratio corresponding to minimal temperature and pressure increase is $2: 1 \sim 2.5: 1$. It also predicted that the ellipticalshaped pin fin structure has the lowest peak temperature and lowest pressure drop. The MOR method was next used to reduce the number of DOFs of large problems with desired the error expectation. The MOR method was applied to the $\mathrm{AD} / \mathrm{DC}$ converter assembly with diamondshaped pin fin heat sinks which was simulated by FEA/CFD. The convective heat transfer coefficients in the MOR model were extracted from the FEA/CFD results. Results show that the MOR results have overall less than 2-6\% deviation from FEA/CFD prediction and the solution takes $<1$ second CPU time. MOR is thus a very effective method for simulation of EV power electronics. The MOR method can be further used with electrical circuit model to predict the coupled electro-thermal performance of the EV power electronics.

\section{References}

[1] http://en.wikipedia.org/wiki/Hybrid_electric_vehicle

[2] M. Anwar et al., "HEV architectures - Power electronics optimization through collaboration subtopic: inverter design and collaboration", SAE conferences, Oct. 2010

[3] J. Drobnik, "Power Electronics Conceptual Study for a Small Urban Electric Vehicle" Proceeding of Hybrid and Fuel Cell Electric Vehicle Symposium and Exhibition (EVS25), China, Nov. 2010.

[4] ANSYS, ANSYS Inc., http://www.ansys.com

[5] A.C. Antoulas, "Approximation of Large-Scale Dynamical Systems". Society for Industrial and Applied Mathematics, 2005, ISBN: 0898715296.

[6] A.C. Antoulas and D.C. Sorensen, "Approximation of large-scale dynamical systems: An overview",
Technical Report, Rice University, Houston, 2001, http://www-ece.rice.edu/ aca/mtns00.pdf.

[7] T. Bechtold, E.B. Rudnyi and J.G. Korvink, "Error indicators for fully automatic extraction of heat transfer macromodels for MEMS", Journal of Micromechanics and Microengineering, 2005, v15, N3, pp. 430-440.

[8] E. Rudnyi, "Effective Electrothermal Simulation for Battery Pack and Power Electronics in HEV/EV", 3rd Virtual Vehicle Symposium in Graz, GSVF, 6-7 May, 2010.

[9] MOR for ANSYS, http://ModelReduction.com

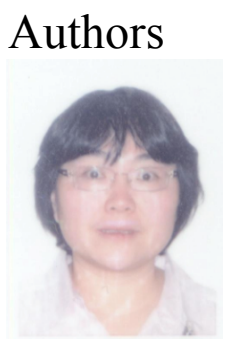

Dr. Wenjun Liu received the BS and MS degree from Tsinghua University, P.R. China in 1997 and 2000 respectively. She received the $\mathrm{PhD}$ degree in Mechanical Engineering at Carnegie Mellon University in 2005. She was a Senior Research Engineer with Intel Cooperation before joining Freescale Semiconductor, where she is now a System and Application Engineer.

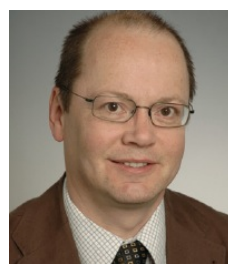

Dr. Torsten Hauck received his diploma in material science from the University Halle and his $\mathrm{PhD}$ degree in fracture mechanics from the University Paderborn, both in Germany. In 1996 he joined Motorola where he worked as senior staff engineer in the semiconductor products sector. Since 2004 he is with the Freescale Semiconductor $\mathrm{GmbH}$ in Munich, Germany, where he is responsible for modelling and simulation of microelectronics components and Microsystems

Dr. Joe Drobnik completed his education (M.Sc \& PhD) in Europe at Czech Technical University of Prague.

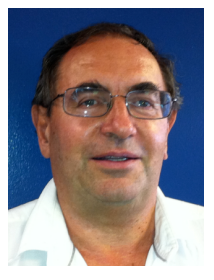

In 1982, during the deepest communistic depression he left Czechoslovakia from political reasons and settled in Canada. In Canada he has been working in the field of Power Electronics with steadily increasing responsibilities. In order to expand his R\&D work, Joe relocated in 1995 to the USA where he worked for Corporate Research of GE, Intel and Freescale. At Freescale Joe leads the technology development for HEV and EV. Joe is a senior member of IEEE. 